\title{
Cooling Trapped Atoms in Optical Resonators
}

\author{
Stefano Zippilli ${ }^{1}$ and Giovanna Morigi ${ }^{2}$ \\ ${ }^{1}$ Abteilung für Quantenphysik, Universität Ulm, D-89069 Ulm, Germany \\ ${ }^{2}$ Departament de Fisica, Universitat Autònoma de Barcelona, 08193 Bellaterra, Spain
}

(Received 25 May 2005; published 28 September 2005)

\begin{abstract}
We derive an equation for the cooling dynamics of the quantum motion of an atom trapped by an external potential inside an optical resonator. This equation has broad validity and allows us to identify novel regimes where the motion can be efficiently cooled to the potential ground state. Our result shows that the motion is critically affected by quantum correlations induced by the mechanical coupling with the resonator, which may lead to selective suppression of certain transitions for the appropriate parameters regimes, thereby increasing the cooling efficiency.
\end{abstract}

DOI: 10.1103/PhysRevLett.95.143001

Cavity cooling is a recent expression, which stresses the role of the mechanical effects of a resonator on the atomic and molecular center-of-mass dynamics. Indeed, the coupling between resonator and atom gives rise to complex dynamics, one aspect of which is the substantial modification of the atom spectroscopic properties [1]. This property allows one to change the atom scattering cross section, thereby affecting, and eventually tailoring, the mechanical dynamics of the atomic center of mass [2]. Moreover, the motion of the atom changes the medium density, thereby affecting the resonator field itself. Several recent experiments have reported relevant features of these complex dynamics [3-8]. Experimental demonstrations of atom cooling in resonators [3-7] have shown, among others, that cavities are a promising tool for preparing and controlling cold atomic samples of scalable dimensions, which may find relevant applications, for instance, in quantum information processing [9].

In this Letter, we present a study of the quantum dynamics of the center-of-mass motion of an atomic dipole, which couples to a resonator and to a laser field driving it from the side. The system is sketched in Fig. 1. Differently from recent theoretical works on cavity cooling of atomic clouds [10], here the center of mass is confined by a tight trap, in a configuration that may correspond to the experimental situations reported, for instance, in $[4,11,12]$. Starting from a master equation for the quantum variables of the dipole, cavity, and center of mass, we derive a closed equation for the center-of-mass dynamics. This equation generalizes previous theoretical studies $[13,14]$ and allows us to identify novel parameter regimes, where cooling can be efficient. Moreover, its form permits us to identify the individual scattering contributions, thereby gaining insight into the role of the various physical parameters. We show that quantum correlations between atom and resonator may lead to the suppression of scattering transitions, thereby enhancing the cooling efficiency.

The starting point is the master equation for an atom of mass $M$ whose dipole transition between the ground and excited states $|g\rangle$ and $|e\rangle$ couples (quasi)resonantly to a laser and the mode of an optical resonator with wave vector
PACS numbers: 32.80.Pj, 32.80.Lg, 42.50.Pq

$k_{L}$ and $k_{c}$, respectively $\left(\left|k_{L}\right| \approx\left|k_{c}\right|=k\right)$. In the reference frame of the laser, the state $|e\rangle$ is at frequency $-\Delta$ and the cavity mode at $-\delta_{c}$. The atomic center of mass is trapped by a harmonic oscillator of frequency $\nu$ along the $x$ axis, while the motion in the orthogonal plane is frozen out. Later on we discuss how the treatment can be generalized to three-dimensional motion. The density matrix $\rho$ of cavity mode and atom internal and external degrees of freedom evolves according to the master equation $\partial \rho / \partial t=\mathcal{L} \rho$, where the Liouvillian $\mathcal{L}$ describes the coherent and incoherent dynamics. External and internal degrees of freedom are coupled through the spatial gradient of the interaction with the electromagnetic field, which scales with the Lamb-Dicke parameter $\eta=\sqrt{\hbar k^{2} / 2 M \nu}$ [15]. We assume tight confinement, such that the gradient of the electromagnetic field over the atomic wave packet is small and the Lamb-Dicke regime holds, $\eta \ll 1$, and treat the coupling of the center of mass with dipole and cavity variables in perturbation theory. At second order in $\eta$ we decompose $\mathcal{L}=\mathcal{L}_{0}+\eta \mathcal{L}_{1}+\eta^{2} \mathcal{L}_{2}+o\left(\eta^{3}\right)$, where the subscripts indicate the corresponding order in the expansion. The term $\mathcal{L}_{0}=\mathcal{L}_{0 E}+\mathcal{L}_{0 I}$ is the sum of the Liouvillian for the external $(E)$ and internal and cavity degrees of freedom $(I)$. Here, $\mathcal{L}_{0 E} \rho=\left[H_{\mathrm{mec}}, \rho\right] / i \hbar$ and

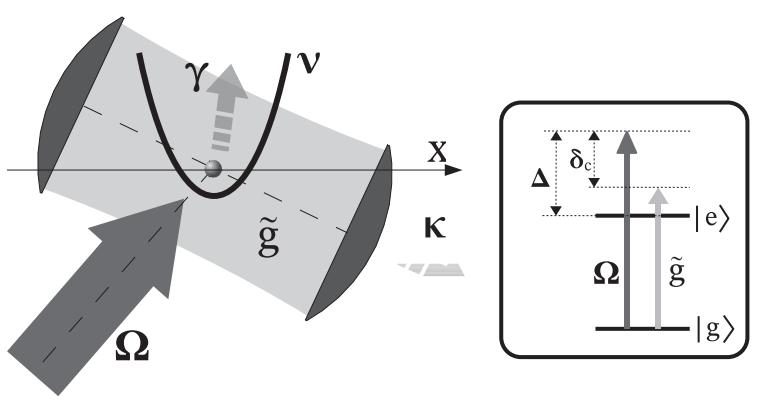

FIG. 1. An atom is confined by an external potential inside an optical resonator and couples to a laser and a mode of the resonator. Energy is dissipated by spontaneous emission, at rate $\gamma$, and by cavity decay, at rate $\kappa$. Inset: Dipole levels $|g\rangle$ and $|e\rangle . \Delta$ and $\delta_{c}$ are the detunings of dipole and cavity from the laser frequency. 
$H_{\text {mec }}=\hbar \nu(\hat{n}+1 / 2)$ center-of-mass harmonic oscillator with $\hat{n}$ number operator for its phononic excitations; the term $\mathcal{L}_{0 I} \rho=\left[H_{0}, \rho\right] / i \hbar+\left(\mathcal{L}_{s}+\mathcal{K}\right) \rho$, where $\mathcal{K}$ and $\mathcal{L}_{s}$ describe cavity decay at rate $\kappa$ and dipole spontaneous emission at rate $\gamma$, respectively [16], and $H_{0}=-\hbar \Delta \sigma^{\dagger} \sigma-$ $\hbar \delta_{c} a^{\dagger} a+\hbar\left[\sigma^{\dagger}(\Omega+\tilde{g} a)+\right.$ H.c. $]$, with $\sigma=|g\rangle\langle e|, a, a^{\dagger}$ annihilation and creation operators of a cavity photon, $\tilde{g}$ coupling constant between cavity and dipole at the center of the trap, and $\Omega$ laser Rabi frequency. The first-order correction $\mathcal{L}_{1} \rho=[V, \rho] / \mathrm{i} \hbar$ contains the operator $V=$ $\left(V_{L}+V_{c}\right)\left(b+b^{\dagger}\right)$, which gives the mechanical coupling induced by laser and resonator, whereby $b, b^{\dagger}$ are the creation and annihilation operators of a vibrational quantum, the operators

$$
\begin{gathered}
V_{L}=i \hbar \varphi_{L} \Omega\left(\sigma^{\dagger}-\sigma\right), \\
V_{c}=\hbar \varphi_{c} \tilde{g}\left(a \sigma^{\dagger}+a^{\dagger} \sigma\right),
\end{gathered}
$$

and the coefficients $\varphi_{L}, \varphi_{c}$ scale the corresponding recoil and depend on the geometry of the setup [17]. The term $\mathcal{L}_{2}$ describes mechanical effects associated with the spontaneous emission of a photon, which determine diffusion, and state-dependent energy shifts $[18,19]$. The modifications induced by these shifts in the cavity field dynamics have been discussed in [20]. In the present treatment, where $\eta \ll 1$, they are high-order corrections to the external potential and hence do not appreciably affect the center-of-mass dynamics.

At second order in the Lamb-Dicke expansion we derive a rate equation for the occupations of the number states $|n\rangle$ of the center-of-mass harmonic oscillator [18,21]. The rate equation is expressed in terms of the heating rate $\Gamma_{n \rightarrow n+1}=\eta^{2}(n+1) A_{+}$and the cooling rate $\Gamma_{n \rightarrow n-1}=$ $\eta^{2} n A_{-}$, which describe transitions that change $|n\rangle$ by one excitation. Here,

$$
A_{ \pm}=\operatorname{Re}\left[2 \operatorname{Tr}_{I}\left\{V\left(\mathcal{L}_{0 I}+i \nu\right)^{-1} V \rho_{0}\right\}+\alpha \gamma \operatorname{Tr}\left\{|e\rangle\langle e| \rho_{0}\right\}\right]
$$

with $\operatorname{Tr}_{I}$ trace over the dipole and cavity degrees of freedom and $\alpha$ geometric factor giving the angular dispersion of atomic momentum due to the random recoil of spontaneous emission. Their explicit evaluation requires the knowledge of the spectrum of the correlation of the operator $V$ and of the steady state $\rho_{0}$ of dipole and cavity mode at zero order in $\eta[18,19,22]$. When the laser weakly perturbs the atom-cavity ground state $\left|\phi_{0}\right\rangle=\left|g, 0_{c}\right\rangle$, we find [21]

$$
\begin{aligned}
A_{ \pm}= & \gamma \alpha\left|\mathcal{T}_{S}\right|^{2}+\gamma\left|\varphi_{L} \mathcal{T}_{L}^{\gamma, \pm}+\varphi_{c} \mathcal{T}_{c}^{\gamma, \pm}\right|^{2} \\
& +\kappa\left|\varphi_{L} \mathcal{T}_{L}^{\kappa, \pm}+\varphi_{c} \mathcal{T}_{c}^{\kappa, \pm}\right|^{2},
\end{aligned}
$$

where

$$
\begin{gathered}
\mathcal{T}_{S}=\Omega \frac{\delta_{c}+i \kappa / 2}{f(0)}, \\
\mathcal{T}_{L}^{\gamma, \pm}=i \Omega \frac{\left(\delta_{c} \mp \nu+i \kappa / 2\right)}{f(\mp \nu)},
\end{gathered}
$$

$$
\begin{gathered}
\mathcal{T}_{L}^{\kappa, \pm}=i \Omega \frac{\tilde{g}}{f(\mp \nu)} \\
\mathcal{T}_{c}^{\gamma, \pm}=-\Omega \frac{\tilde{g}^{2}\left(2 \delta_{c} \mp \nu+i \kappa\right)}{f(0) f(\mp \nu)}, \\
\mathcal{T}_{c}^{\kappa, \pm}=-\Omega \frac{\tilde{g}\left[(\Delta \mp \nu+\mathrm{i} \gamma / 2)\left(\delta_{c}+i \kappa / 2\right)+\tilde{g}^{2}\right]}{f(0) f(\mp \nu)},
\end{gathered}
$$

with

$$
f(x)=\left(x+\delta_{c}+i \kappa / 2\right)(x+\Delta+i \gamma / 2)-\tilde{g}^{2} .
$$

Equations (3) and (4)-(8) are the main results of this Letter. They have been derived for one-dimensional motion. However, since at second order in $\eta$ the three directions of oscillation decouple in an anisotropic trap, they can be generalized to three-dimensional motion as they hold for any geometry of the setup. Their analytic form allows insight into these complex dynamics, which we now discuss.

The rates are the incoherent sum of three contributions. The first term on the right-hand side (rhs) of Eq. (3), $\gamma \alpha\left|\mathcal{T}_{S}\right|^{2}$, describes absorption of a laser photon and spontaneous emission, whereby the change in the center-ofmass state is due to the recoil induced by the spontaneously emitted photon. This process is shown in Fig. 2(a), where the excitation paths are depicted in the dressed states basis $| \pm ; n\rangle$ of cavity and atom, which are superpositions of the states $\left|g, 1_{c}\right\rangle$ and $\left|e, 0_{c}\right\rangle$ [2] at a fixed number of phonons $|n\rangle$. The scattering rate is scaled by the geometric factor $\alpha$ and is found after averaging over the solid angle of photon emission into free space. This contribution is diffusive, as the motion can be scattered into a higher or lower vibrational state with probabilities depending on the overlap integrals [19]. Diffusion can be suppressed, namely, term (4) can become very small, in good resonators, $\kappa \ll \tilde{g}$, and for $\delta_{c}=0$. In this limit the corresponding dynamics are characterized by destructive interference between the two excitation paths $\left|\phi_{0} ; n\right\rangle \rightarrow| \pm ; n\rangle[23-25]$. This situation appears at zero order in the mechanical effects and is reminiscent of analogous phenomena encountered in the cooling dynamics of trapped multilevel atoms [26].

The second term on the rhs of Eq. (3), $\gamma \mid \varphi_{L} \mathcal{T}_{L}^{\gamma, \pm}+$ $\left.\varphi_{c} \mathcal{T}_{c}^{\gamma, \pm}\right|^{2}$, describes scattering of a laser photon by spontaneous emission where the motion is changed by mechanical coupling to the laser $\left(\mathcal{T}_{L}^{\gamma, \pm}\right)$ and to the cavity $\left(\mathcal{T}_{c}^{\gamma, \pm}\right)$ field. Process $\mathcal{T}_{L}^{\gamma, \pm}$ scales with the geometric factor $\varphi_{L}$, which accounts for the recoil due to absorption of a laser photon, and is depicted in Fig. 2(b). The transition amplitude $\mathcal{T}_{c}^{\gamma, \pm}$, shown in Fig. 2(c), describes the mechanical coupling between the dressed states $| \pm\rangle$ due to the resonator. It thus scales with the geometric factor $\varphi_{c}$ which accounts for the recoil due to interaction with the cavity mode. Since the final state of the two scattering processes is the same, they interfere [27]. In addition, each term is composed by multiple excitation paths and can vanish in some parameter regimes. A representative case 


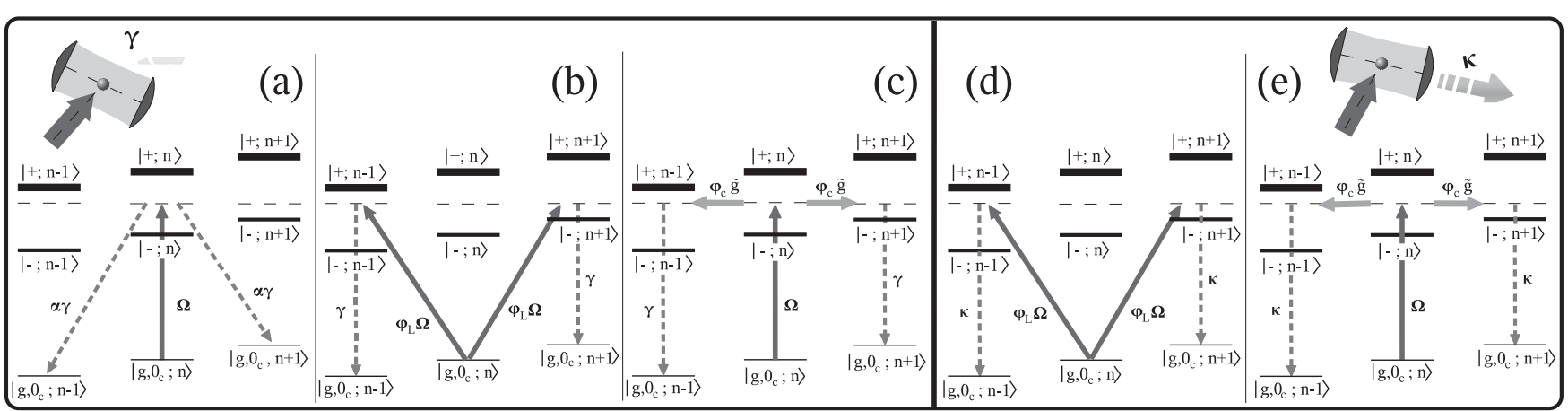

FIG. 2. Scattering processes leading to a change of the vibrational number by one phonon. The states $\left|g, 0_{c} ; n\right\rangle,| \pm ; n\rangle$ are the cavityatom dressed states at phonon number $n$. Processes (a)-(c) describe scattering of a laser photon by spontaneous emission. They prevail in good resonators, for $\kappa \ll \tilde{g}$, $\gamma$. Here, (a) $\mathcal{T}_{S}$; (b) $\mathcal{T}_{L}^{\gamma, \pm}$; (c) $\mathcal{T}_{c}^{\gamma, \pm}$. Processes (d) and (e) describe scattering of a laser photon by cavity decay. They prevail in bad resonators, for $\gamma \ll \tilde{g}$, $\kappa$. Here, (d) $\mathcal{T}_{L}^{\kappa, \pm}$; (e) $\mathcal{T}_{c}^{\kappa, \pm}$. See text.

is found in good resonators, $\kappa \ll \tilde{g}$, when the laser is orthogonal to the motion axis $\left(\varphi_{L}=0\right)$. Then, the transition amplitude, $\mathcal{T}_{c}^{\gamma,+}$, is suppressed for $\delta_{c}=\nu / 2$, as one can see in Eq. (7). This suppression arises from destructive interference between the multiple paths of mechanical coupling via the dressed states $| \pm ; n\rangle$ and $| \pm ; n+1\rangle$, thereby giving a vanishing heating rate. Similarly, the cooling transition can be suppressed by choosing $\delta_{c}=-\nu / 2$.

The third term on the rhs of Eq. (3), $\kappa \mid \varphi_{L} \mathcal{T}_{L}^{\kappa, \pm}+$ $\left.\varphi_{c} \mathcal{T}_{c}^{\kappa, \pm}\right|^{2}$, describes scattering of a laser photon by cavity decay, where the motion is changed by mechanical coupling to the laser $\left(\mathcal{T}_{L}^{\kappa, \pm}\right)$ and to the cavity $\left(\mathcal{T}_{c}^{\kappa, \pm}\right)$ field. The scattered photon is transmitted through the cavity mirrors into the external modes, and therefore these two processes do not interfere with the ones discussed above. They are depicted in Figs. 2(d) and 2(e) and add up coherently with one another. An interesting situation has been discussed in [13], where it was shown that interference between these two terms can lead to suppression of the heating transition in bad resonators. The result of [13] is recovered from Eqs. (3)-(8) in the corresponding parameter regime [21].

The general dynamics are a competition of all these processes. By inspection of the form of Eqs. (3)-(9), one can identify a strategy for obtaining efficient cooling by searching for the parameters such that $\operatorname{Re}\{f(\nu)\}=0$, thereby minimizing the denominator of $A_{-}$, and therefore enhancing the cooling rate over the heating rate. This condition leads to the equation

$$
\Delta_{\mathrm{opt}}\left(\delta_{c}\right) \equiv \frac{\tilde{g}^{2}+\gamma \kappa / 4}{\delta_{c}+\nu}-\nu,
$$

which relates the cavity detuning $\delta_{c}$ to the atom detuning $\Delta$ for fixed couplings and decay rates, such that, when $\Delta=$ $\Delta_{\text {opt }}\left(\delta_{c}\right)$, the cooling rate $A_{-}$is maximum. In general, Eq. (10) sets the best parameters for ground-state cooling, provided that either $\gamma \ll \nu$ or $\kappa \ll \nu$. Below we discuss cooling in the good cavity limit. The bad cavity limit will be discussed elsewhere. Figures 3(a) and 3(b) are contour plots, displaying the average number of phonon at steady state, $\langle n\rangle_{\infty}=A_{+} /\left(A_{-}-A_{+}\right)$, and the cooling rate, $W=$ $\eta^{2}\left(A_{-}-A_{+}\right)$, as a function of $\Delta$ and $\delta_{c}$ for a good cavity and $\kappa \ll \nu \ll \gamma$, namely, when processes in Fig. 2(a)2(c) are dominant. These curves have been obtained from Eq. (3), and their validity has been checked by comparison with full numerical simulations [21]. The dashed lines in Figs. 3(a) and 3(b) indicate the curve $\Delta=\Delta_{\text {opt }}\left(\delta_{c}\right)$. It is evident that this curve corresponds to the parameter region where the lowest temperature is achieved. The final limit is set by the ratio $\kappa / \nu$, since in this regime $\kappa$ is the narrowest linewidth one can achieve for the scattering processes [28].

Good parameter regimes are found for relatively small values of $\Delta$ and $\delta_{c}$, in the range $0<\delta_{c}<\nu, \Delta>0$, where one has low temperatures and relatively large cooling rates for a wide range of values. These regions correspond to novel cooling dynamics, which are characterized by suppression of heating and diffusive transitions by quantum correlations induced by the resonator. Here, for instance,
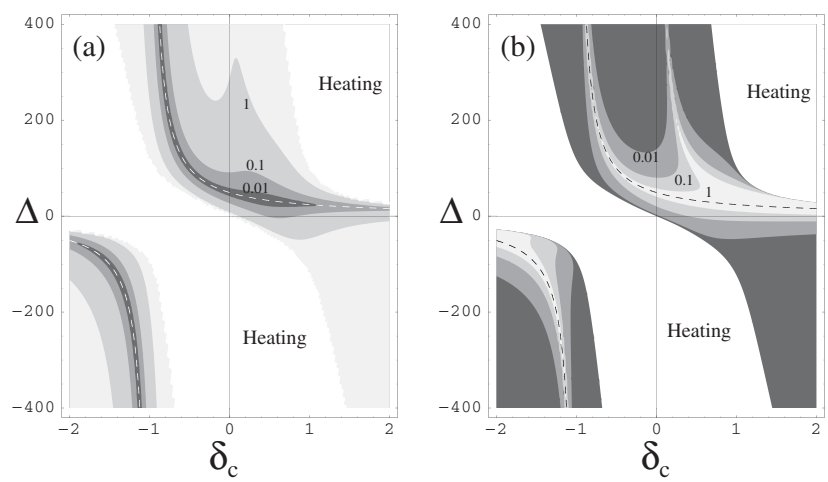

FIG. 3. Contour plot of (a) the average phonon number at steady state $\langle n\rangle_{\infty}$ and (b) the corresponding cooling rate $W$ (units of $10^{-3} \nu$ ) as a function of $\delta_{c}$ and $\Delta$ (units of $\nu$ ) in the good cavity limit. The darkest region corresponds to the smallest values, the lightest region to the largest values. The numbers indicate the values at the corresponding contour line. The heating regions are not coded and explicitly indicated. The dashed lines represent Eq. (10). Here, $\eta=0.1, \varphi_{L}=\varphi_{c}=$ $\sqrt{1 / 2}, \Omega=\nu, \tilde{g}=7 \nu, \gamma=10 \nu$, and $\kappa=0.01 \nu$. 
the potential ground-state occupation can reach $99 \%$ in a time $\tau \sim 2 \mathrm{~ms}$ for an atom in a trap with frequency $\nu=$ $2 \pi \times 500 \mathrm{KHz}$ and $\eta=0.1$, whose dipole has linewidth $\gamma=2 \pi \times 5 \mathrm{MHz}$ and couple to a resonator with $g=$ $2 \pi \times 5 \mathrm{MHz}$ and $\kappa=2 \pi \times 100 \mathrm{KHz}$. Larger values of $\kappa$ lead to smaller occupations, which scale as $\langle n\rangle_{\infty} \sim$ $\kappa^{2} / \nu^{2}$ in good resonators for $\kappa<\nu$. Figure 3 shows also that the dynamics are relatively insensitive to fluctuations of the parameters in the range $0<\delta_{c}<\nu, \Delta>0$. This implies that several oscillation modes, such as the ones of an ion chain [29], could be simultaneously cooled. We remark that good cooling regions are found in Fig. 3 also for large $|\Delta|$ and $\delta_{c} \sim-\nu$. This is the so-called cavity sideband cooling regime, discussed in [14]. Here, enhancement of the cooling rate is achieved by means of resonance with the very narrow transition, at large $|\Delta|$, between the dressed states of the cavity-atom coupling. This regime exhibits a critical sensitivity to variations of the value of $\delta_{c}$ with respect to $\Delta$.

To conclude, we have presented the explicit form of cooling and heating rates of the center-of-mass motion of an atom, trapped in an optical resonator and driven by a laser. This result permits us to identify the basic processes determining the center-of-mass dynamics and the parameter regions where cooling can be efficiently obtained. Novel regimes have been identified, where the motion is cooled by exploiting destructive interference in the multiple paths of the mechanical excitations. The resulting interference bases itself on the discreteness of the vibrational spectrum, which is the same for the dipolar ground and the excited state. Some analogies with interference processes in the cooling of trapped multilevel atoms $[26,30]$ are due to a similar dressed state structure at low excitation; nevertheless the mechanical effects induced by the resonator give rise to a wider wealth of phenomena. Dynamics will be substantially modified when the external potential depends appreciably on the internal state $[20,22]$. In future works, we will investigate how the dynamics are modified by collective effects, when many atoms are trapped inside the cavity.

Beyond applications to cooling, these results allow one to gain insight into the complex dynamics of the mechanical effects of optical resonators on atoms, and their wealth of phenomena could be exploited for implementing coherent control of this kind of system.

We thank Helmut Ritsch and Axel Kuhn for discussions. Support from the IST-network QGATES and the Spanish Ministerio de Educación y Ciencia (Ramon-y-Cajal Grant No. 129170) is acknowledged.

[1] See, for instance, Cavity Quantum Electrodynamics, edited by P. R. Berman (Academic Press, New York, 1994).
[2] P. Domokos and H. Ritsch, J. Opt. Soc. Am. B 20, 1098 (2003).

[3] P. Maunz et al., Nature (London) 428, 50 (2004).

[4] J. McKeever et al., Phys. Rev. Lett. 90, 133602 (2003).

[5] H. W. Chan, A. T. Black, and V. Vuletic, Phys. Rev. Lett. 90, 063003 (2003); A. T. Black, H. W. Chan, and V. Vuletic, Phys. Rev. Lett. 91, 203001 (2003).

[6] D. Kruse et al., Phys. Rev. Lett. 91, 183601 (2003); B. Nagorny, T. Elsasser, and A. Hemmerich, Phys. Rev. Lett. 91, 153003 (2003).

[7] C. von Cube et al., Phys. Rev. Lett. 93, 083601 (2004).

[8] P. Bushev et al., Phys. Rev. Lett. 92, 223602 (2004).

[9] See, for instance, The Physics of Quantum Information, edited by D. Bouwmeester, A. K. Ekert, and A. Zeilinger (Springer-Verlag, Berlin, 2000).

[10] P. Domokos and H. Ritsch, Phys. Rev. Lett. 89, 253003 (2002); P. Domokos, Th. Salzburger, and H. Ritsch, Phys. Rev. A 66, 043406 (2002); P. Domokos, A. Vukics, and H. Ritsch, Phys. Rev. Lett. 92, 103601 (2004).

[11] M. Keller et al., Nature (London) 431, 1075 (2004); A. B. Mundt et al., Phys. Rev. Lett. 89, 103001 (2002).

[12] J. A. Sauer et al., Phys. Rev. A 69, 051804(R) (2004).

[13] J. I. Cirac, M. Lewenstein, and P. Zoller, Phys. Rev. A 51, 1650 (1995).

[14] V. Vuletic, H. W. Chan, and A. T. Black, Phys. Rev. A 64, 033405 (2001).

[15] S. Stenholm, Rev. Mod. Phys. 58, 699 (1986).

[16] H. J. Carmichael, An Open Systems Approach to Quantum Optics (Springer-Verlag, Berlin, 1993).

[17] For instance, $\varphi_{L}=\cos \theta$ when the laser is a traveling wave forming the angle $\theta$ with the axis of the motion.

[18] J. Javanainen, M. Lindberg, and S. Stenholm, J. Opt. Soc. Am. B 1, 111 (1984).

[19] J. I. Cirac et al., Phys. Rev. A 46, 2668 (1992).

[20] J. Leach and P.R. Rice, Phys. Rev. Lett. 93, 103601 (2004).

[21] S. Zippilli and G. Morigi, quant-ph/0508075.

[22] G. Morigi, Phys. Rev. A 67, 033402 (2003).

[23] P. M. Alsing, D. A. Cardimona, and H. J. Carmichael, Phys. Rev. A 45, 1793 (1992).

[24] S. Zippilli, G. Morigi, and H. Ritsch, Phys. Rev. Lett. 93, 123002 (2004); Eur. Phys. J. D 31, 507 (2004).

[25] P. R. Rice and R. J. Brecha, Opt. Commun. 126, 230 (1996).

[26] G. Morigi, J. Eschner, and C. H. Keitel, Phys. Rev. Lett. 85, 4458 (2000); J. Evers and C. H. Keitel, Europhys. Lett. 68, 370 (2004).

[27] They also interfere with the amplitude $\mathcal{T}_{S}$. This interference depends on the angle of emission in free space and is thus averaged out after integrating over the solid angle.

[28] Lowest occupations can be reached by using a standing wave drive [21].

[29] G. Morigi and Sh. Fishman, Phys. Rev. Lett. 93, 170602 (2004).

[30] I. Marzoli et al., Phys. Rev. A 49, 2771 (1994). 